Nonlinear Mathematical Physics 1996, V.3, N 1-2, 186-195.

\title{
Second-Order Differential Invariants for Some Extensions of the Poincaré Group and Invariant Equations
}

\author{
Irina YEHORCHENKO \\ Institute of Mathematics, National Academy of Sciences of Ukraïna, \\ 3 Tereshchenkivs'ka Street, Kÿ̈v 4, Ukrä̈na
}

\begin{abstract}
It is well-known that symmetry properties are extremely important for choosing differential equations which can be suitable for description of real physical processes.

We present functional bases of second-order differential invariants for various representations of some extensions of the Poincaré group and for a set of $m$ scalar functions (e.g., for similarity and conformal groups). These results enable us to describe new classes of nonlinear multi-dimensional invariant equations and to simplify the problem of symmetry classification of second-order scalar partial differential equations.
\end{abstract}

\section{Introduction}

The concept of invariant is widely used in various domains of mathematics. In this paper we investigate differential invariants within the framework of symmetry analysis of differential equations.

Differential invariants and construction of invariant equations were considered by S. Lie [1] and his followers [2, 3]. Tresse [2] proved the theorem on existence and finiteness of a functional basis of differential invariants. However, there exist few papers devoted to construction of differential invariants for specific groups involved in mechanics and mathematical physics in explicit form.

Knowledge of differential invariants of a certain algebra or group facilitates classification of equations invariant with respect to this algebra or group. There are also some general methods for investigation of differential equations which need the explicit form of differential invariants for symmetry groups of these equations' (see, e.g., [3, 4]).

A brief review of our investigation of second-order differential invariants for the Poincaré and Galilei groups is given in $[5,6]$. Our results on functional bases of differential invariants are founded on Lemma about functionally independent invariants for the proper orthogonal group and for two $n$-dimensional symmetric tensors of order 2 [7].

We should like to stress that we consider functionally independent invariants of tensors but not irreducible ones, as in the classical theory of invariants.

Bases of irreducible invariants for the group $O(3)$ and three-dimensional symmetric tensors and vectors are adduced in [8].

DEFINITION 1. A maximal set of functionally independent invariants of order $r \leq l$ of the Lie algebra $L$ is called a functional basis of the $l$ th-order differential invariants for the algebra $L$. 
We consider invariants of order 1 and 2, and we need the first and second prolongations of the operator

$$
X=\xi^{i} \partial_{x_{i}}+\eta^{r} \partial_{u^{r}}
$$

(see, e.g., $[9-12])$

$$
\stackrel{1}{X}=X+\eta_{i}^{r} \partial_{u_{i}^{r}}, \quad \stackrel{2}{X}=\stackrel{1}{X}+\eta_{i j}^{r} \partial_{u_{i j}^{r}},
$$

the coefficients $\eta_{i}^{r}$ and $\eta_{i j}^{r}$ taking the form

$$
\begin{aligned}
& \eta_{i}^{r}=\left(\partial_{x_{i}}+u_{i}^{s} \partial_{u^{s}}\right) \eta^{r}-u_{k}^{r}\left(\partial_{x_{i}}+u_{i}^{s} \partial_{u^{s}}\right) \xi^{k} \\
& \eta_{i j}^{r}=\left(\partial_{x_{i}}+u_{j}^{s} \partial_{u^{s}}+u_{j^{k}}^{s} \partial_{u_{k}^{s}}\right) \eta_{i}^{r}-u_{i k}^{r}\left(\partial_{x_{j}}+u_{j}^{s} \partial_{u^{s}}\right) \xi^{k} .
\end{aligned}
$$

While writing out lists of invariants, we shall use the following designations

$$
\begin{aligned}
& u_{a} \equiv \frac{\partial u}{\partial x_{a}}, \quad u_{a b} \equiv \frac{\partial^{2} u}{\partial x_{a} \partial x_{b}}, \\
& S_{k}\left(u_{a b}\right) \equiv u_{a_{1} a_{2}} u_{a_{2} a_{3}} \cdots u_{a_{k-1} a_{k}} u_{a_{k} a_{1}}, \\
& S_{j k}\left(u_{a b}, v_{a b}\right) \equiv u_{a_{1} a_{2}} \cdots u_{a_{j-1} a_{j}} v_{a_{j} a_{j+1}} \cdots v_{a_{k} a_{1}}, \\
& R_{k}\left(u_{a}, u_{a b}\right) \equiv u_{a_{1}} u_{a_{k}} u_{a_{1} a_{2}} u_{a_{2} a_{3}} \cdots u_{a_{k-1} a_{k}} .
\end{aligned}
$$

Here and further we mean summation over repeated indices from 1 to $n$. In all lists of invariants, $k$ takes values from 2 to $n$ and $j$ takes values from 0 to $k$. We shall not discern upper and lower indices with respect to summation: for all Latin indices

$$
x_{a} x_{a} \equiv x_{a} x^{a} \equiv x^{a} x_{a}=x_{1}^{2}+x_{2}^{2}+\cdots+x_{n}^{2} .
$$

When we calculate second prolongations with the usual Lie technique (see e.g. [9]), we imply that action of an operator of the form $X^{a b} \partial_{u_{a b}}$, where $X^{a b}$ are some functions, is as follows:

$$
X^{a b} \partial_{u_{a b}}\left(u_{c d} u_{c d}\right)=2 X^{a b} u_{a b} ; \quad \partial_{u_{a b}} u_{c d}=\delta_{a c} \delta_{b d} .
$$

With this assumption, $\partial_{u_{a b}} u_{b a}=0, a \neq b$.

\section{Differential Invariants for the Euclid Algebra}

We consider the Euclid algebra $A E(n)$ defined by the basis operators

$$
\partial_{a} \equiv \frac{\partial}{\partial x_{a}}, \quad J_{a b}=x_{a} \partial_{b}-x_{b} \partial_{a} .
$$

Here and further, the letters $a, b, c, d$, when used as indices, take values from 1 to $n, n$ being the number of space variables $(n \geq 3)$.

The algebra $A E(n)$ is an invariance algebra for a wide class of multi-dimensional scalar equations involved in mathematical physics - the Schrödinger, heat, d'Alembert equations, etc. 
In this section we shall explain in detail how to construct a functional basis of secondorder differential invariants for the algebra $A E(n)$. This basis will be used further to find invariant bases for various algebras containing the Euclid algebra as a subalgebra.

\subsection{MAIN RESULTS}

THEOREM 1. There is a functional basis of second-order differential invariants for the Euclid algebra $A E(n)$ with the basis operators (3) for a scalar function $u=u\left(x_{1}, \ldots, x_{n}\right)$ consisting of these $2 n+1$ invariants

$$
u, \quad S_{k}\left(u_{a b}\right), \quad R_{k}\left(u_{a}, u_{a b}\right) .
$$

THEOREM 2. Second-order differential invariants of the algebra $A E(n)$ (1.1) for a set of scalar functions $u^{r}, r=1, \ldots, m$, can be represented as functions of the following expressions:

$$
u^{r}, \quad S_{j k}\left(u_{a b}^{1}, u_{a b}^{r}\right), \quad R_{k}\left(u_{a}^{r}, u_{a b}^{1}\right) .
$$

Proofs of these theorems can be found in [7].

\subsection{BASES OF INVARIANTS FOR THE EXTENDED EUCLID ALGEBRA AND CONFORMAL ALGEBRA}

The extended Euclid algebra $A E_{1}(n)$ for one scalar function is defined by the basis operators $\partial_{a}, J_{a b}(3)$ and $D$ depending on a parameter $\lambda$ :

$$
D=x_{a} \partial_{a}+\lambda u \partial_{u}\left(\partial_{u}=\frac{\partial}{\partial u}\right) .
$$

The basis of the conformal algebra $A C(n)$ consists of the operators $\partial_{a}, J_{a b}(3), D(6)$ and

$$
K_{a}=2 x_{a} D-x_{b} x_{b} \partial_{a} .
$$

THEOREM 3. There is a functional basis for the extended Euclid algebra that has the following form

(1) when $\lambda \neq 0$ :

$$
\frac{R_{k}\left(u_{a}, u_{a b}\right)}{u^{k(1-2 / \lambda)+1}}, \quad \frac{S_{k}\left(u_{a b}\right)}{u^{k(1-2 / \lambda)}} ;
$$

(2) when $\lambda=0$ :

$$
u, \quad \frac{R_{k}\left(u_{a}, u_{a b}\right)}{\left(u_{a a}\right)^{k}}, \quad \frac{S_{k}\left(u_{a b}\right)}{\left(u_{a a}\right)^{k}} \quad(k \neq 1) ;
$$

a functional basis for the conformal algebra has the following form:

(1) when $\lambda \neq 0$ :

$$
S_{k}\left(\theta_{a b}\right) u^{k(2 / \lambda-1)},
$$

(2) when $\lambda=0$ :

$$
u, S_{k}\left(w_{a b}\right)\left(u_{a} u_{a}\right)^{-2 k} \quad(k \neq n),
$$


where

$$
\begin{aligned}
& \theta_{a b}=\lambda u_{a b}+(1-\lambda) \frac{u_{a} u_{b}}{u}-\delta_{a b} \frac{u_{c} u_{c}}{2 u} \\
& w_{a b}=u_{c} u_{c}\left(u_{a b}+\frac{\delta_{a b}}{2-n} u_{d d}\right)-u_{c}\left(u_{a} u_{b c}+u_{b} u_{a c}\right),
\end{aligned}
$$

$\delta_{a b}$ being the Kronecker symbol.

Proofs of Theorem 3 and all theorems below can be performed using the standard Lie algorithm $[9,10]$.

DEFINITION 2. Tensors $\theta_{a}$ and $\theta_{a b}$ of order 1 and 2 are called covariant with respect to some algebra $L=\left\langle J_{a b}, X_{i}\right\rangle$ if

$$
\begin{aligned}
X_{i} \theta_{a} & =\sigma_{a b}^{i} \theta_{b}+\sigma^{i} \theta_{a} \\
X_{i} \theta_{a b} & =\rho_{a c}^{i} \theta_{c b}+\rho_{b c}^{i} \theta_{a c}+\rho^{i} \theta_{a b}
\end{aligned}
$$

$X_{i}$ are operators of the form (1), $\rho^{i}, \sigma^{i}$ are some functions, $\sigma_{a b}^{i}, \rho_{a b}^{i}$ are some skewsymmetric tensors.

It is easy to show that the expressions $S_{k}\left(\theta_{a b}\right), R_{k}\left(\theta_{a}, \theta_{a b}\right)$, where $\theta_{a}, \theta_{a b}$ are tensors covariant with respect to the algebra $L$, are relative invariants of this algebra.

The fact that $\theta_{a b}$ and $w_{a b}(12)$ are covariant with respect to the conformal algebra $A C(n)$ can be verified by direct substitution of these tensors into the invariance conditions for the operators $\stackrel{2}{D}$ and $\stackrel{2}{K_{a}}$.

Note 1. When $\lambda \neq 0$, covariant tensors exist for $A C(n)$ of order 2 only; when $\lambda=0$, the tensors $w_{a b}(12)$ and $u_{a}$ are conformally covariant but $S_{k}\left(w_{a b}\right)$ and $R_{k}\left(u_{a}, w_{a b}\right)$ are dependent.

THEOREM 4. Second-order differential invariants for a vector function $u=\left(u^{1}, \ldots u^{m}\right)$ and for the algebra $A E_{1}(n)=\left\langle\partial_{a}, J_{a b}, D\right\rangle$, the operator $D$ having the form

$$
D=x_{a} \partial_{a}+\lambda u^{r} \partial_{u^{r}}
$$

with summation over $r$ from 1 to $m$, can be represented as functions of the following expressions:

(1) when $\lambda \neq 0$ :

$$
\frac{u^{r}}{u^{1}} \quad(r=2, \ldots, m), \quad \frac{S_{j k}\left(u_{a b}^{1}, u_{a b}^{r}\right)}{\left(u^{1}\right)^{k(1-2 / \lambda)}}, \quad \frac{R_{k}\left(u_{a}^{r}, u_{a b}^{1}\right)}{\left(u^{1}\right)^{k(1-2 / \lambda)+1}} ;
$$

(2) when $\lambda=0$ :

$$
u^{r}, \quad R_{k}\left(u_{a}^{r}, u_{a b}^{1}\right) \cdot\left(u_{a a}^{1}\right)^{-k}, \quad S_{j k}\left(u_{a b}^{1}, u_{a b}^{r}\right) \cdot\left(u_{a a}^{1}\right)^{-k}
$$

(when $r=1$ then $k \neq 1$ );

the corresponding basis for the conformal algebra $A C(n)=\left\langle\partial_{a}, J_{a b}, D, K_{a}\right\rangle$, where

$$
K_{a}=2 x_{a} D-x_{b} x_{b} \partial_{a}
$$

has the following form: 
(1) when $\lambda \neq 0$ :

$$
S_{j k}\left(\theta_{a b}^{r}, \theta_{a b}^{1}\right)\left(u^{1}\right)^{k(2 / \lambda-1)}, \frac{u^{r}}{u^{1}}, R_{k}\left(\theta_{a}^{r}, \theta_{a b}^{1}\right)\left(u^{1}\right)^{k(2 / \lambda-1)-1} \quad(r=2, \ldots, m) ;
$$

(2) when $\lambda=0$ :

$$
u^{r}(r=1, \ldots, m),\left(u_{d}^{1} u_{d}^{1}\right)^{-2 k} S_{j k}\left(w_{a b}^{1}, w_{a b}^{r}\right), \quad\left(u_{d}^{1} u_{d}^{1}\right)^{-2 k} R_{k}\left(u_{a}^{r}, w_{a b}^{1}\right) \quad(r=2, \ldots, m)
$$

(for the set of invariants $\left(u_{d}^{1} u_{d}^{1}\right)^{-2 k} S_{k}\left(w_{a b}\right), k$ does not take the value $n$ ); the tensors $\theta_{a b}^{r}, w_{a b}^{r}$ are constructed similarly to (13) and

$$
\theta_{a}^{r}=\frac{u_{a}^{r}}{u^{r}}-\frac{u_{a}^{1}}{u^{1}}
$$

\subsection{DIFFERENTIAL INVARIANTS OF THE ROTATION ALGEBRA}

The rotation algebra is defined by basis operators $J_{a b}(3)$.

Second-order invariants of this algebra for $m$ scalar functions $u^{r}$ are constructed with $x_{a}, u^{r}, u_{a}^{r}, w_{a b}$ similarly to invariants of the Euclid algebra.

THEOREM 5. There is a functional basis of second-order differential invariants for the algebra $A O(n)$ that has the form

$$
u^{r}, \quad S_{j k}\left(u_{a b}^{1}, u_{a b}^{r}\right), \quad R_{k}\left(u_{a}^{r}, u_{a b}^{1}\right), \quad R_{k}\left(x_{a}, u_{a b}^{1}\right) ; \quad r=1, \ldots, m ;
$$

the corresponding basis of invariants for the algebra $\left\langle J_{a b}, D\right\rangle$, where $D$ is defined by (14), consists of the expressions

$$
\begin{aligned}
& \frac{u^{r}}{u^{1}} \quad(r=2, \ldots, m), \quad \frac{S_{j k}\left(u_{a b}^{1}, u_{a b}^{r}\right)}{\left(u^{1}\right)^{k(1-2 / \lambda)},} \\
& R_{k}\left(u_{a}^{r}, u_{a b}^{1}\right) \cdot\left(u^{1}\right)^{2 k / \lambda-1-k}, \\
& R_{k}\left(x_{a}, u_{a b}^{1}\right) \cdot\left(u^{1}\right)^{2 / \lambda(k-2)-k+1}, \quad \text { when } \lambda \neq 0 ; \\
& u^{r}, R_{k}\left(u_{a}^{r}, u_{a b}^{1}\right) \cdot\left(u_{a a}^{1}\right)^{-k}, \\
& S_{j k}\left(u_{a b}^{1}, u_{a b}^{r}\right) \cdot\left(u_{a a}^{1}\right)^{-k} \quad(k \neq 1 \text { when } r=1), \\
& R_{k}\left(x_{a}, u_{a b}^{1}\right) \cdot\left(u_{a a}^{1}\right)^{2-k} \quad \text { when } \lambda=0 .
\end{aligned}
$$

$A$ basis of invariants for the algebra $\left\langle J_{a b}, D, K_{a}\right\rangle$, when $\lambda \neq 0$, consists of the expressions (15) and

$$
\frac{R_{k}\left(x_{a}, \theta_{a b}^{1}\right)}{x^{2}\left(u^{1}\right)^{(k-1)(1-2 / \lambda)}}, \quad k=2, \ldots, n+1
$$

when $\lambda=0$, it consists of the expressions (16) and

$$
\frac{R_{k}\left(x_{a}, w_{a b}^{1}\right)}{x^{2}\left(w_{a a}^{1}\right)^{k-1}} \quad\left(x^{2}=x_{a} x_{a}\right)
$$




\section{Differential Invariants of the Poincaré and Conformal Algebra}

In this section we consider differential invariants of the second order for a set of $m$ scalar functions

$$
u^{r}=u^{r}\left(x_{0}, x_{1}, \ldots, x_{n}\right), \quad n \geq 3 .
$$

The Poincaré algebra $A P(1, n)$ is defined by the basis operators

$$
\begin{aligned}
p_{\mu} & =i g_{\mu \nu} \frac{\partial}{\partial x_{\nu}}, \\
J_{\mu \nu} & =x_{\mu} p_{\nu}-x_{\nu} p_{\mu},
\end{aligned}
$$

where $\mu, \nu$ take values $0,1, \ldots, n$; summation is implied over repeated indices (if they are small Greek letters) in the following way:

$$
\begin{aligned}
& x_{\nu} x_{\nu} \equiv x_{\nu} x^{\nu} \equiv x^{\nu} x_{\nu}=x_{0}^{2}-x_{1}^{2}-\cdots-x_{n}^{2}, \\
& g_{\mu \nu}=\operatorname{diag}(1,-1, \ldots,-1) .
\end{aligned}
$$

We consider $x_{\nu}$ and $x^{\nu}$ equivalent with respect to summation not to mix signs of derivatives and numbers of functions.

Quasilinear second-order invariants of the Poincaré algebra were described in [13].

THEOREM 6. There is a functional basis of second-order differential invariants of the Poincaré algebra $A P(1, n)$ for a set of $m$ scalar functions $u^{r}$ consisting of

$$
m(2 n+3)+(m-1) \frac{n(n+1)}{2}
$$

invariants

$$
u^{r}, \quad R_{k}\left(u_{\mu}^{r}, u_{\mu \nu}^{1}\right), \quad S_{j k}\left(u_{\mu \nu}^{r}, u_{\mu \nu}^{1}\right) .
$$

In this section, everywhere $k=1, \ldots, n+1 ; j=0, \ldots, k ; r=1, \ldots, m$.

For the extended Poincaré algebra $A \tilde{P}(1, n)=\left\langle p_{\mu}, J_{\mu \nu}, D\right\rangle$, where

$$
D=x_{\mu} p_{\mu}+\lambda u^{r} p_{u^{r}}
$$

$\left(p_{u^{r}}=i\left(\partial / \partial u^{r}\right)\right.$, summation over $r$ from 1 to $m$ is implied $)$ the corresponding basis has the following form:

(1) when $\lambda=0$ :

$$
u^{r}, S_{j k}\left(u_{\mu \nu}^{r}, u_{\mu \nu}^{1}\right) \cdot\left(u_{\alpha \alpha}^{1}\right)^{-k}
$$

(2) when $\lambda \neq 0$ :

$$
\frac{u^{r}}{u^{1}}, \quad S_{j k}\left(u_{\mu \nu}^{r}, u_{\mu \nu}^{1}\right)\left(u^{1}\right)^{2 k / \lambda-k-1}, \quad R_{k}\left(u_{\mu}^{r}, u_{\mu \nu}^{1}\right)\left(u^{1}\right)^{k(2 \lambda-1)},
$$

where $S_{j k}, R_{k}$ are defined similarly to (2) and summation over small Greek indices is of the type (18). 
For the conformal algebra $A C(1, n)=\left\langle p_{\mu}, J_{\mu \nu}, D, K_{\mu}\right\rangle$, where

$$
K_{\mu}=2 x_{\mu} D-x_{\nu} x_{\nu} p_{\mu}
$$

( $D$ being the dilation operator (19)), the corresponding basis consists of the expressions

$$
S_{j k}\left(\theta_{\mu}^{r}, \theta_{\mu \nu}^{1}\right) \cdot\left(u^{1}\right)^{k(2 / \lambda-1)}, \quad \frac{u^{r}}{u^{1}}, \quad R_{k}\left(\theta_{\mu}^{r}, \theta_{\mu \nu}^{1}\right) \cdot\left(u^{1}\right)^{k(2 / \lambda-1)-1} ;
$$

when $\lambda \neq 0 ; r=2, \ldots, m$, there is no summation over $r$; conformally covariant tensors have the form

$$
\begin{aligned}
\theta_{\mu}^{r} & =\frac{u_{\mu}^{r}}{u^{r}}-\frac{u_{\mu}^{1}}{u^{1}}, \\
\theta_{\mu \nu}^{r} & =\lambda u_{\mu \nu}^{r}+(1-\lambda) \frac{u_{\mu}^{r} u_{\nu}^{r}}{u^{r}}-g_{\mu \nu} \frac{u_{\beta}^{r} u_{\beta}^{r}}{2 u^{r}}
\end{aligned}
$$

When $\lambda=0$, the corresponding basis of invariants for the conformal algebra has the form

$$
\begin{aligned}
& u^{r}, \quad S_{j k}\left(w_{\mu \nu}^{r}, w_{\mu \nu}^{1}\right) \cdot\left(u_{\alpha}^{1} u_{\alpha}^{1}\right)^{-2 k}, \\
& R_{k}\left(u_{\mu}^{r}, w_{\mu \nu}^{1}\right) \cdot\left(u_{\alpha}^{1} u_{\alpha}^{1}\right)^{1-2 k}, \quad r=2, \ldots, m ;
\end{aligned}
$$

the tensors $\left(w_{\mu \nu}^{r}\right)$,

$$
w_{\mu \nu}^{r}=u_{\alpha}^{r} u_{\alpha}^{r}\left(u_{\mu \nu}^{r}-\frac{g_{\mu \nu}}{1-n} u_{\beta \beta}^{r}\right)-u_{\beta}^{r}\left(u_{\mu}^{r} u_{\beta \nu}^{r}+u_{\nu}^{r} u_{\beta \mu}^{r}\right)
$$

are conformally invariant (there is no summation over $r$ ).

Similarly to the results of Section 1.4, it is possible to construct invariants of the algebras $\left\langle J_{\mu \nu}\right\rangle,\left\langle J_{\mu \nu}, D\right\rangle,\left\langle J_{\mu \nu}, D, K_{\mu}\right\rangle$.

The obtained results allow us to construct new nonlinear multi-dimensional equations, e.g., the equation

$$
\frac{u_{\alpha} u_{\alpha}}{1-n} u_{\nu \nu}-u_{\mu} u_{\nu} u_{\mu \nu}=\left(u_{\nu} u_{\nu}\right)^{2} F(u)
$$

where $F$ is an arbitrary function, is invariant under the algebra $A C(1, n), \lambda=0$.

There is another quasi-linear relativistic equation with rich symmetry properties

$$
\left(1-u_{\alpha} u_{\alpha}\right) u_{\mu \mu}-u_{\alpha} u_{\mu} u_{\alpha \mu}=0,
$$

that is the Born-Infeld equation. Symmetry and solutions of this equation were investigated in $[11,14]$. This equation is invariant under the algebra $A P(1, n+1)$ with the basis operators

$$
J_{A B}=x_{A} p_{B}-x_{B} p_{A}, \quad A, B=1, \ldots, n+1 ; \quad x_{n+1} \equiv u .
$$

Let us consider a class of equations

$$
u_{\mu \nu} u_{\mu \nu}=F\left(u_{\mu \mu}, u_{\mu} u_{\nu} u_{\mu \nu}, u_{\mu} u_{\mu}, u\right) .
$$


It is evident that they are invariant with respect to the Poincaré algebra $A P(1, n)$ but a straightforward search for conformally invariant equations from this class with the standard Lie technique requires a lot of cumbersome calculations. Using of differential invariants turns this problem into one of elementary algebra, e.g., if $\lambda \neq 0$,

$$
F-u_{\mu \nu} u_{\mu \nu}=-\frac{1}{\lambda} S_{2}\left(\theta_{\mu \nu}\right)+u^{2(1-2 / \lambda)} \phi\left(S_{1}\left(\theta_{\mu \nu}\right) u^{2 / \lambda-1}\right)
$$

where $\theta_{\mu \nu}$ is of the form (1.24) and $\phi$ is an arbitrary function. Whence

$$
\begin{aligned}
F= & u^{2(1-2 / \lambda)} \phi\left(u^{2 / \lambda-1}\left(u_{\mu \mu}-\frac{\lambda+n}{\lambda} \frac{u_{\alpha} u_{\alpha}}{u}\right)\right)- \\
& \frac{1}{\lambda^{2} u^{2}}\left(\lambda^{2}+n^{2}\right)\left(u_{\alpha} u_{\alpha}\right)^{2}-\frac{2(1-\alpha)}{\lambda u} u_{\mu} u_{\nu} u_{\mu \nu}+\frac{2 u_{\mu \mu} u_{\alpha} u_{\alpha}}{\lambda u} .
\end{aligned}
$$

It is useful to note that besides traces of matrix powers (3), one can utilize all possible invariants of covariant tensors $\theta_{\mu \nu}^{r}, w_{\mu \nu}^{r}$ to construct conformally invariant equations.

\section{Dif ferential Invariants of an Infinite-Dimensional Algebra}

It is well-known that the simplest first-order relativistic equation - the eikonal or Hamilton equation

$$
u_{\alpha} u_{\alpha} \equiv u_{0}^{2}-u_{1}^{2}-\cdots-u_{n}^{2}=0
$$

is invariant under the infinite-dimensional algebra $A P^{\infty}(1, n)$ generated by the operators $[11,15]$

$$
X=\left(b^{\mu \nu} x_{\nu}+a^{\mu}\right) \partial_{\mu}+\eta \partial_{u},
$$

$-b^{\mu \nu}=b^{\nu \mu}, a^{\mu}, \eta$ being arbitrary differentiable functions of $u$. Equation (20) is widely used in geometrical optics.

In this section, we describe a class of second-order equations invariant under the algebra (21).

It is easy to show that the tensors

$$
\begin{aligned}
\theta_{\mu} & =\frac{u_{\mu}}{\left(u_{\alpha} u_{\alpha}\right)^{1 / 2}}, \\
\theta_{\mu \nu} & =\frac{u_{\mu \nu} u_{\beta} u_{\beta}+u_{\beta \beta} u_{\mu} u_{\nu}-u_{\beta} u_{\beta \mu} u_{\nu}-u_{\beta} u_{\beta \nu} u_{\mu}}{\left(u_{\alpha} u_{\alpha}\right)^{3 / 2}}
\end{aligned}
$$

are covariant under the algebra $A P^{\infty}(1, n)(21)$.

THEOREM 7. The equations of the form

$$
S_{k}\left(\theta_{\mu \nu}\right)=0, \quad k=1,2, \ldots,
$$

$S_{k}$ being defined as (2), are invariant with respect to the algebra $A P^{\infty}(1, n)(21)$.

The problem of description of all such equations is more difficult and we do not consider it here.

Convolutions of the form

$$
R\left(\theta_{\mu}, \theta_{\mu \nu}\right)
$$


appear to be functionally dependent on the above.

Let us investigate in more detail a quasi-linear second-order equation of the form

$$
u_{\mu} u_{\mu \nu} u_{\nu}-u_{\mu} u_{\mu} u_{\alpha} u_{\alpha}=0 .
$$

THEOREM 8. When $n \geq 2$, Equation (24) is invariant with respect to the algebra $A \tilde{P}^{\infty}(1, n)$ with generators of the form

$$
X+d(u) x_{\mu} p_{\mu},
$$

$X$ is of the form (21), $d(u)$ is an arbitrary function of $u$.

Let us present another example of the $A \tilde{P}^{\infty}(1, n)$-invariant equation:

$$
u_{\mu \nu} u_{\mu \nu} \cdot u_{\beta} u_{\beta}-2 u_{\mu} u_{\beta \nu} u_{\mu \nu} u_{\beta}-(\square u)^{2} u_{\beta} u_{\beta}=0 .
$$

\section{Conclusion}

It is well-known that a mathematical model of physical or some other phenomenon must obey one of the relativity principles of Galilei or Poincaré. Speaking the language of mathematics, we can say that it means that equations of the model must be invariant under the Galilei or the Poincaré groups. Having bases of differential invariants for these groups (or for the corresponding algebras), we can describe all invariant scalar equations, or sort the invariant ones out of a set of equations.

Construction of differential invariants for vector and spinor fields presents more complicated problems. First-order invariants for a four-dimensional vector potential were found in [16]. The cases of spinor and multi-dimensional vector Poincaré-invariant equations and corresponding bases of invariants are still to be investigated.

\section{Acknowledgment}

The author acknowledges financial support by DKNT of Ukraina (project N 11.3./42). 


\section{References}

[1] Lie S., Math. Ann., 1884, V.24, 52-89.

[2] Tresse A., Acta Math., 1894, V.18, 1-88.

[3] Vessiot E., Acta Math., 1904, V.28, 307-349.

[4] Michal A.D., Proc. Nat. Acad. Sci., 1951, V.37, 623-627.

[5] Fushchych W.I. and Yehorchenko I.A., Dokl. AN Ukr. SSR, Ser. A, 1989, N 4, 29-32.

[6] Fushchych W.I. and Yehorchenko I.A., Dokl. AN Ukr. SSR, Ser. A, 1989, N 5, 21-22.

[7] Fushchych W.I., Yegorchenko I.A., Acta Appl. Math., 1992, V.28, 69-92.

[8] Spencer A.J.M., Theory of Invariants, Academic Press, New York, London, 1971.

[9] Ovsyannikov L.V., Group Analysis of Differential Equations, Academic Press, New York, 1982.

[10] Olver P., Application of Lie Groups to Differential Equations, Springer-Verlag, New York, 1987.

[11] Fushchych W.I., Shtelen W.M. and Serov, N.I., Symmetry Analysis and Exact Solutions of NonLinear Equations of Mathematical Physics, Naukova Dumka, Kiev, 1989 (in Russian; English version - Kluwer Publishers, 1993).

[12] Bluman G.W. and Kumei S. Symmetries and Differential Equations, Springer Verlag, New York, 1989.

[13] Fushchych W.I. and Yehorchenko, I.A., Dokl. AN SSSR, 1988, V.298, 347-351.

[14] Fushchych W.I. and Serov N.I., Dokl. AN SSSR, 1984, V.278, 847.

[15] Fushchych W.I. and Shtelen W. M., Lett. Nuovo Cimento, 1982, V.34, 498.

[16] Yehorchenko I.A., Symmetry properties of nonlinear equations for complex vector fields, Preprint 89.48, Institute of Mathematics of the Ukr. Acad. Sci., 1989. 